\title{
Portrait statistique de l'écrivain journaliste en Belgique francophone entre 1918 et 1960
}

Une certaine doxa affirme que le journalisme permet à l'écrivain de vivre de sa plume. C'est en tout cas la recette que beaucoup semblent avoir choisie dans la première moitié du $x^{\mathrm{e}}$ siècle en Belgique francophone. Si la polygraphie des écrivains belges a été étudiée par Sophie Piron à propos des genres littéraires ${ }^{1}$, elle ne se limite pas à ce domaine : nombreux sont les écrivains qui produisent des textes non littéraires ou, pour le dire plus précisément, qui n'ont pas la littérarité pour objectif premier. Nous avons constaté précédemment qu'à la fin du XIX et au début du $\mathrm{XX}^{\mathrm{e}}$ siècles, les juristes - autre grande catégorie socioprofessionnelle entretenant un lien étroit avec l'écrit - représentaient une part non négligeable des auteurs belges francophones ${ }^{2}$. Dans cet article, nous étudierons la présence des journalistes au sein de l'institution littéraire : qui sont-ils ? qu'écrivent-ils ? où publientils ? où s'exerce leur activité journalistique ? quelle est la part du journalisme dans leurs activités professionnelles?

À dire vrai, cependant, poser ces questions, c'est déjà préjuger d'une réalité sociale particulièrement mouvante. Comme d'autres articles de ce dossier l'ont souligné, le journaliste est un travailleur qu'il est difficile de cerner. La durée de sa participation à un périodique ne nous renseigne pas sur les revenus qu'il en tire. Certains exhibent fièrement cette profession, que d'autres dissimulent comme si elle était honteuse. Enfin, le monde de la presse est particulièrement

1 Piron (Sophie), "La polygraphie chez les écrivains belges au début du $X X^{\mathrm{e}}$ siècle », dans Klinkenberg (Jean-Marie), dir, Textyles, n²15, L'Institution littéraire, 1998, pp. 86-101.

2 Dozo (Bjön-Olav), "La présence des juristes dans l'institution littéraire belge. Examen de l'évolution des trajectoires scolaires et professionnelles des écrivains dans l'entre-deuxguerres », dans Brogniez (Laurence), dir, Textyles, n³1, Droit et littérature, 2007, p. 28 46. 
propice aux investissement éphémères ou de circonstance : plus que toute autre, la profession de journaliste peut être exercée pendant un temps limité, de manière intensive, ou être reprise après une longue absence. C'est pourquoi la catégorie spécifique de « journaliste » est peu opératoire dans une étude statistique. Nous avons préféré construire une catégorie plus large au départ, celle des « professions de l'information, des arts et des spectacles » 3 , abrégéé « professions IAS », que nous allons explorer, en signalant ce qui est exclusivement spécifique aux journalistes quand il le sera possible.

La base du CIEL ${ }^{4}$ répertorie ainsi 669 auteurs ayant publié au moins un ouvrage littéraire entre 1918 et 1960 . Elle recense en outre la date de naissance et la (ou les) profession(s) de 522 auteurs parmi les 669. Ces 522 auteurs ont exercé 253 professions différentes (dans l'état actuel de la base). Parmi ces professions, nous en avons catalogué 638 comme professions IAS, catégorie qui comprend principalement des journalistes et que nous allons examiner plus en détail. Enfin, sur 522 auteurs, 225 ont exercé une « profession de l'information, des arts et des spectacles $\gg$ (soit $43 \%$ ), alors qu'ils ne sont que 89 (soit un peu plus de $17 \%$ ) sur la période ayant exercé une profession libérale (c'est-à-dire les avocats et assimilés et, dans une faible proportion, les médecins).

Cette catégorie des professions IAs rassemble donc une série d'écrivains qui n'ont pas été journalistes du tout : 48 écrivains sur les 225 (soit $21,3 \%$ ) regroupés dans la catégorie. Les professions ainsi désignées sont, principalement, « homme ou femme de lettres » (catégorie floue, qui pourrait cacher des journalistes; nous y reviendrons à propos de la question des «écrivains professionnels »), « peintre», « directeur de théâtre », « conservateur de musée », «éditeur ». Si l'on examine la présence des différentes professions du journalisme (étiquettes « journaliste » et ses déclinaisons : « reporter», « collaborateur », « rédacteur », etc.), on dénombre 120 professions sur les 638 répertoriées dans la catégorie, soit $18,8 \%$. Le journalisme y reste fortement majoritaire : il est représenté par 518 professions, soit plus de $81 \%$ du total de la catégorie.

Ainsi, il nous semble pertinent de conserver cette catégorie des professions IAS en l'état, sans en isoler les journalistes, car ceux-ci y sont de toute manière très largement majoritaires. En outre, la catégorie de l'« écrivainjournaliste » reste ambiguë vu l'absence de définition formalisée du métier de « journaliste »

Nous avons déjà évoqué plus haut la difficile question des critères de définition de la profession de journaliste. Revenons-y plus en détail. Différentes pistes de réflexion pourraient être envisagées, selon différents critères (ceux

3 Pour le détail de la nomenclature socioprofessionnelle utilisée, voir ibidem, pp. 36-39.

4 Le Collectif interuniversitaire d'étude du littéraire développe une base de données sur les auteurs, les œuvres et les revues littéraires belges francophones entre 1918 et 1920. Elle est accessible à l'adresse 〈http://www.cicl-litterature.be> qui suivent ne sont pas exhaustifs, mais visent seulement à illustrer notre propos) : le critère économique, qui définirait comme écrivain-journaliste tout écrivain qui gagnerait sa vie en produisant de la copie (mais que faire des journalistes amateurs ?) ; le critère de production (avoir publié un certain nombre - mais lequel ? - d'articles dans la presse - mais de quel type ?) ; le critère de sociabilité professionnelle (l'appartenance à une association professionnelle de journalistes garantirait le statut, mais que faire des auteurs abondamment publiés dans les journaux qui n'appartiennent à aucune de ces associations ?) ; etc. Ingrid Mayeur, dans le présent numéro, dresse la liste des écrivains-journalistes qu'elle a pu constituer à partir du dépouillement des annuaires professionnels. En partant de la pratique journalistique (et donc de listes de journalistes établies à partir des annuaires et du Dictionnaire des journalistes-écrivains de Belgique de Lionel Bertelson ${ }^{5}$ ), elle a dû par la suite « opérer une sélection et écarter les journalistes n'ayant publié aucun écrit à caractère littéraire durant l'intervalle de temps retenu » ${ }^{6}$. De notre côté, nous sommes partis d'une liste d'écrivains (celle de la base CIEL) et avons vérifié leurs professions, de sorte que, des journalistes-écrivains, nous passons aux écrivains-journalistes. Ces deux perspectives sont complémentaires et aboutissent, finalement, à l'enrichissement et à l'exploitation de la base de données du CIEL.

Il nous semble cependant nécessaire de mettre en évidence et d'exploiter cette différence de choix méthodologiques dans la constitution du corpus. Ingrid Mayeur part de la sociabilité professionnelle, qu'elle complète grâce à un critère de production littéraire. Pour notre part, notre corpus repose sur une liste d'écrivains ayant fait un choix professionnel leur permettant de vivre d'une source de revenus proche du régime vocationnel de l'écrivain. En d'autres termes, le journalisme partage avec la profession d'écrivain, d'une part, un rapport à la culture et, d'autre part, une certaine forme d'écriture créative. On retrouve cette manifestation créative, dans des proportions diverses, au sein des autres professions regroupées dans cette catégorie (acteur, peintre, homme de lettres, etc.) ${ }^{7}$. À partir de là, sans nous restreindre à l'étude des écrivainsjournalistes, c'est la situation économique de la profession d'écrivain, particulièrement les aménagements et compromis possibles avec d'autres

5 Bertelson (Lionel), Dictionnaire des Journalistes-Écrivains de Belgique, Bruxelles, Association générale de la presse de Belgique, 1960.

6 MaYeur (Ingrid), «Les journalistes écrivains belges (1920-1960) 》, dans la présente livraison.

7 La catégorie des professions IAS ne contient pas une autre profession créative, celle d'« enseignant-chercheur », qui bénéficie d'une catégorie spécifique dans la nomenclature socioprofessionnelle que nous avons retenue. Cette différence se marque par un rapport à la création ne répondant pas aux mêmes buts et exigences. Évidemment, ces catégories sont le fruit d'un héritage socioculturel complexe, que bien des pratiques peuvent remettre en question, mais qui nous semblent suffisamment éprouvées pour rendre raison de manière
générale de notre nhjet. 
formes de créativité plus rentables, qu'il nous est permis d'interroger. L'étude de la catégorie des professions IAS constitue donc un moyen de réfléchir à cette situation en élargissant le point de vue.

Notre étude consistera davantage en une exploration de cette catégorie qu'en une comparaison de l'évolution des professions des écrivains pour la période donnée. C'est que la proportion de ces professions parmi les écrivains semble énorme et reste constante de génération en génération.

Si l'on examine la proportion de «professions IAS » parmi les autres professions en fonction des classes d'âge des écrivains, on obtient le graphique suivant :

Figure 1 - Professions « journaliste » par classes d'âge

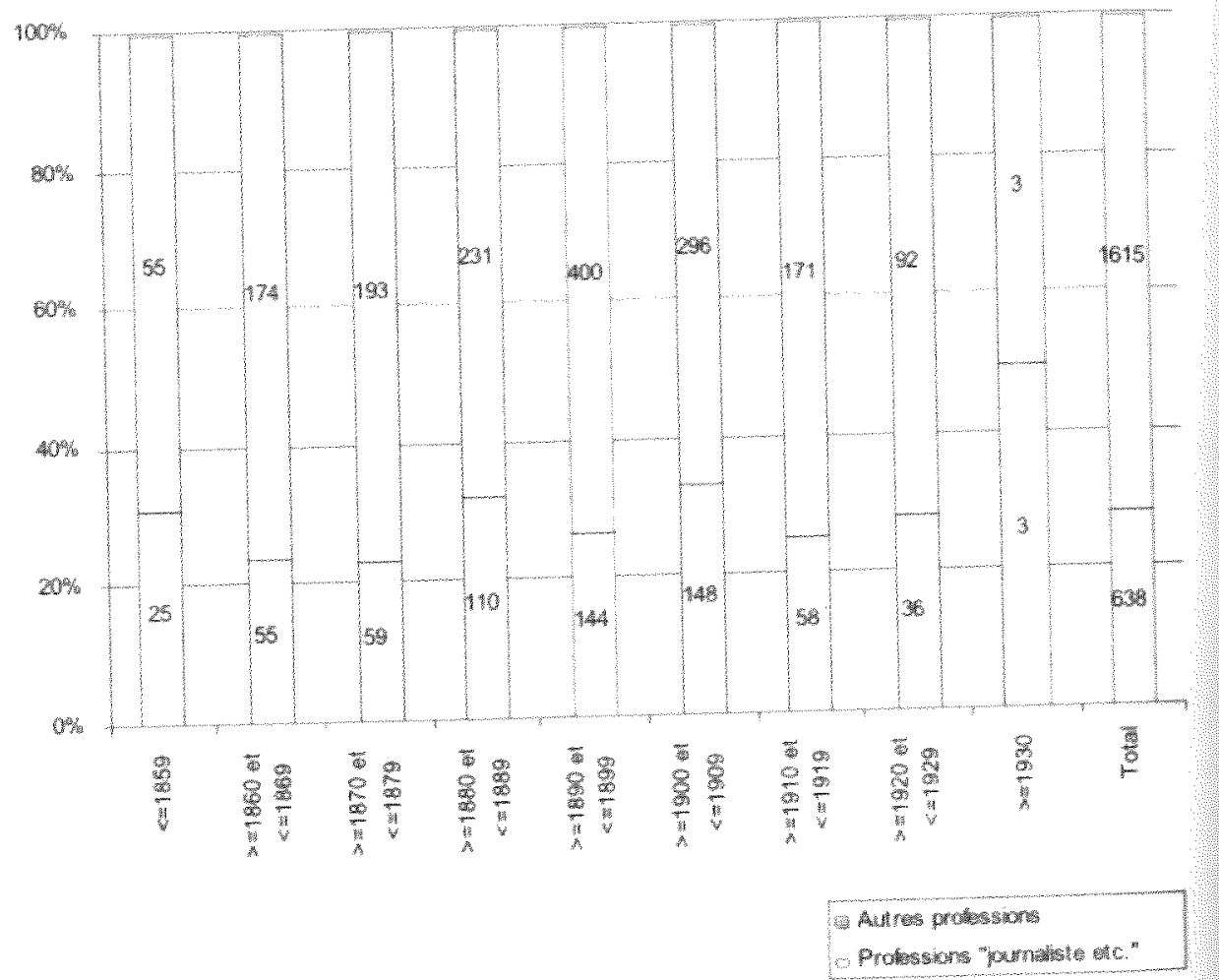

On constate que cette proportion est forte pour toutes les classes d'âge. Elle oscille entre 25 et $50 \%$ des professions exercées par les écrivains selon la classe d'âge. Si l'on examine le nombre d'écrivains qui ont exercé une profession de ce type par classes d'âge, la tendance se confirme
Figure 2 - Journalistes par classes d'âge

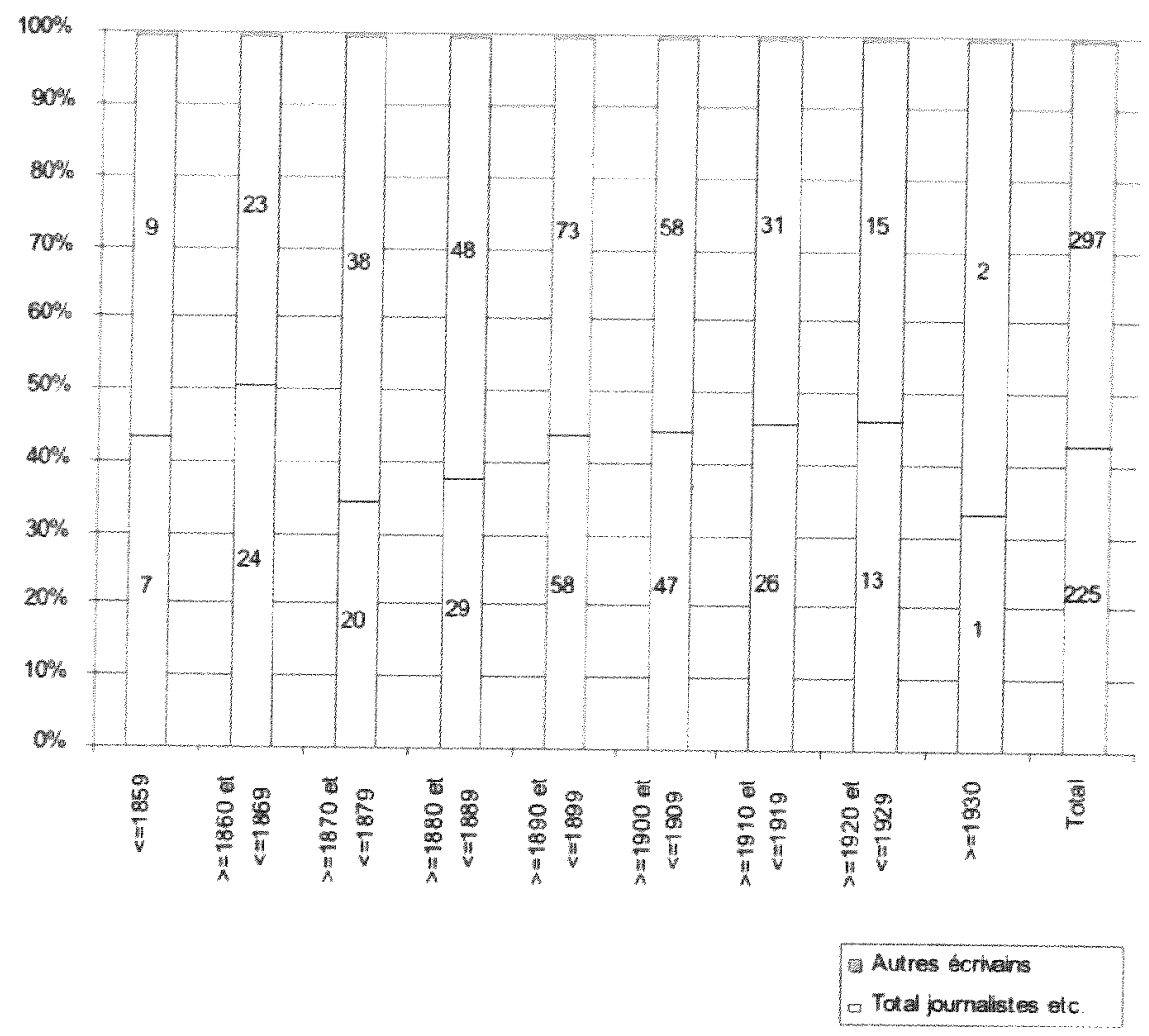

Quelle que soit sa date de naissance, plus d'un écrivain belge actif durant l'entre-deux-guerres sur trois (voire presque un sur deux pour certaines classes d'âge) a été journaliste ou assimilé durant sa vie. La proportion est donc importante ramenée aux individus eux-mêmes, plus encore que celle que l'on a obtenue en restant au niveau des professions diverses des écrivains.

Cette présence massive semble donc confirmer la doxa énoncée au début de cet article. Néanmoins, l'évidence de cette activité chez les littérateurs est telle qu'elle a été peu étudiée. Il nous faut donc l'interroger.

\section{Mise à l'épreuve du corpus : le sous-corpus féminin}

Avant tout, il faut mesurer les conséquences des choix de critères qui ont présidé à la constitution de notre corpus, en particulier l'usage de la catégorie " professions IAS », plus large que celle de journalistes. Pour mesurer ce que 
la catégorie recouvre, comparons le corpus ainsi défini à celui de Vanessa Gemis ${ }^{8}$, qui étudie les femmes écrivaines journalistes sur la même période.

Vingt-cinq écrivaines sont incluses dans notre corpus des auteurs ayant exercé une profession de l'information, des arts et des spectacles. Quinze sont communes à la liste isolée par Vanessa Gemis (Adine, Augis, Burniaux, Cappe, De Smet, Goldstein, Hamoir, Hermendes, Lilar, Migeon, Piette, Revelard, Sneyers, Van de Wiele, Wannyn). Trois femmes, considérées comme des écrivaines-journalistes, s'ajoutent par rapport à la liste de comparaison. Elles ont pu échapper au premier repérage par manque d'informations les concernant: dans la base ciEL, Simone Berson est renseignée comme journaliste, sans plus de précision, Jean(ni)ne Gosselin est critique cinématographique et Margherite Inghels a participé aux journaux d'extrême-droite Rex, Le Pays réel et Voilà 9 .

A la différence du critère de constitution, il faut ajouter une différence de catégorisation : la catégorie des "professions IAS» est plus vaste que la catégorie « journaliste », qu’elle englobe. La catégorisation large prend en compte les formes de journalisme oral, même quand celui-ci est la seule forme de journalisme exercée par l'écrivain : par exemple, le joumalisme télévisuel, avec Françoise Mallet-Joris, chroniqueuse pour l'émission française "Panorama chrétien " ${ }^{10}$. Mais la catégorisation large entraine des scories par rapport à une catégorie focalisée uniquement sur le journalisme : les peintres Marie Howet et Emma Lambotte, la lectrice pour les éditions Laffont Marie de Vivier et les femmes de lettres Béatrix Beck, Marie Gevers et Marguerite Yourcenar font partie du corpus des professions IAS. Si les trois premières ne peuvent être considérées comme journalistes, cela paraît moins net pour les trois dernières : l'étiquette «femmes de lettres » peut cacher toute activité de plume; le journalisme pourrait en faire partie.

La catégorie des professions IAs déplace donc légèrement l'angle de vue mais permet d'englober des réalités comparables (notamment un rapport au littéraire, voire à l'artistique, comme source de revenus) qu'une définition plus stricte aurait exclues a priori

crivains journalistes (1880-1940) : questions de genre(s) Pistes de recherche et réflexions autour de Marguerite Van de Wiele ", dans la présente livraison.

9 Ben DiafFar (Lamya), «Les femmes et l'Ordre nouveau en Belgique francophone, 1936$1945 »$, dans Cahiers d'histoire du temps présent, n०4, 1998, p. 149, note 25.

10 Mallet-Joris ne semble pas avoir exercé de journalisme écrit. En revanche, Marc Augis et Estelle Goldstein, que nous avons évoquées plus haut comme journalistes de presse écrite, ont aussi exercé à la radio : Marc Augis a travaillé à l'inR et à Radio-Namur et Estelle Goldstein était journaliste à l'INR.

\section{Les professions de l'information, des arts et des spectacles comme professions complémentaires ou épisodiques}

La catégorie des écrivains-journalistes est sujette par nature à l'hybridation professionnelle. En outre, la pratique journalistique dans la première moitié du $\mathrm{XX}^{\mathrm{e}}$ siècle chez les écrivains belges francophones conforte et amplifie encore cette situation hybride : le journalisme n'est pas une profession constante dans les trajectoires de ces écrivains; elle intervient ponctuellement au cours de leur vie, qui combine bien souvent d'autres professions.

Notre corpus de 225 écrivains exercent 638 professions IAS et, de manière complémentaire au cours de leur vie, 615 autres professions, qu'il s'agit d'examiner.

Durant sa vie, environ un écrivain sur trois exerce uniquement une profession de l'information, des arts et des spectacles (63 écrivains sur 225 exercent ce type de profession de manière exclusive, soit $28 \%$ ). Plus des deux tiers gagnent donc leur vie par d'autres biais. Voici un graphique des autres catégories professionnelles investies par ces écrivains

\section{Autes professions exerces par les ecrivainsjoumalistes au cours de leur vie}

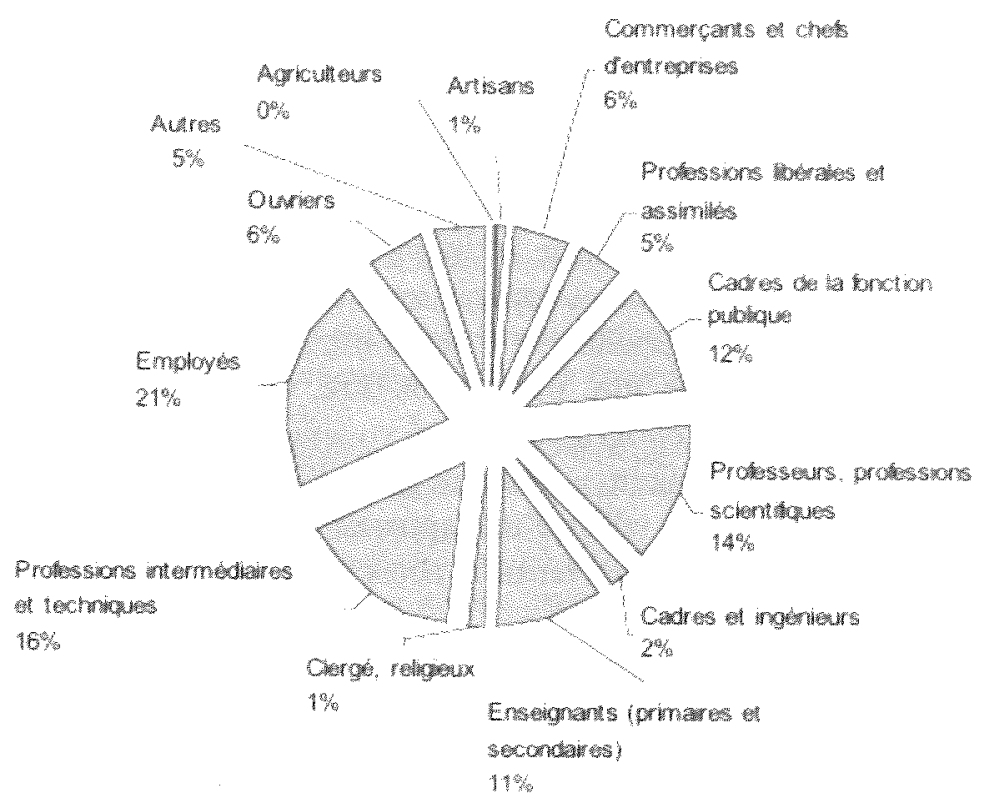

Les métiers de l'IAs semblent donc être les formes courantes de profession complémentaire de la «classe moyenne » des écrivains : de nombreux employés, enseignants et fonctionnaires exercent en plus de leur premier métier celui d'écrivain-journaliste ou assimilé. Se dessine ici une pratique 
du journalisme comme métier irrégulier, assez loin de l'image des grands reporters professionnels qui peuplent un certain imaginaire littéraire.

\section{Sociabilité des écrivains-journalistes}

Un mot sur les lieux de sociabilité de ces écrivains-journalistes indépendamment de la sociabilité spécifique des associations de presse, il est intéressant de remarquer que les écrivains-journalistes développent avant tout une sociabilité littéraire classique en Belgique francophone : 39 sont membres de l'Académie royale de Langue et Littérature françaises de Belgique (ARLLF), 97 de l'Association des Écrivains belges, 29 des Scriptores Catholici, 13 sont signataires du Manifeste du Lundi, etc. On retrouve des membres de tous les lieux de sociabilité belges francophones classiques de l'après-Première Guerre mondiale, jusqu'à plusieurs signataires de tracts surréalistes. Bref, l'écrivainjournaliste, par sa sociabilité, est avant tout un écrivain.

\section{Quelle littérature publient les écrivains-journalistes?}

Venons-en aux genres littéraires pratiqués par ces écrivains. La base de données du CIEL procède d'une approche pragmatique des genres littéraires l'objectif est, d'une part, de recenser la manière dont les genres sont mobilisés dans ce que Gérard Genette a appelé le paratexte (notamment quel genre apparaît sur la couverture de l'ouvrage) ${ }^{11}$ et, d'autre part, de collationner les genres mobilisés par l'histoire de la littérature pour décrire les œuvres.

Pour cette raison, les genres apparaissent sous deux catégories dans la base de données : «genre explicite » et « genre implicite». Le genre est explicite s'il est spécifié dans le titre ou le sous-titre, ou s'il est mentionné entre parenthèses dans la Bibliographie des écrivains francophones de Belgique. Le champ «genre explicite » contient donc l'information littérale, c'est-àdire la mention du genre telle qu'elle apparaît dans les sources. Par exemple, si le titre mentionne «pièce en trois actes », le champ " genre explicite » contiendra «pièce en trois actes ».

Le genre est implicite s'il n'est pas spécifié dans le titre, mais qu'une source extérieure livre l'information (en particulier les catalogues de bibliothèques), ou s'il est mentionné entre crochets dans la Bibliographie des écrivains francophones de Belgique.

Dans cet article, nous ne ferons pas de distinction entre les genres explicites et implicites : ce qui nous intéresse est la mention du genre, peu importe la source pourvu qu'elle soit fiable. La base de données répertorie à

11 Genette (Gérard), Seuils, Paris, Seuil, 1987. l'heure actuelle 9056 éditions de livres écrits par un auteur belge francophone entre 1918 et 1960. Sur ce total, 3391 n'ont pas de mention de genre, soit un peu plus d'un tiers de la production.

Pour pouvoir étudier les mentions de genres de manière sérielle, nous les avons regroupées en grandes catégories. Si les mentions traditionnelles sont les plus nombreuses (roman, poésie, essai, etc.), il apparaît également une myriade de petits genres que nous avons rassemblés soit sous la bannière d'un grand genre, soit en créant une catégorie jugée pertinente.

Nous avons retenu dix genres (biographie, conférences, contes, essai, non littéraire ${ }^{12}$, nouvelles ${ }^{13}$, poésie, roman, témoignages et théâtre, à quoi s'ajoute la catégorie « sans genre » ${ }^{14}$ ) et observé leur évolution sur la période étudiée. (v. fig. 3 p. 132)

Outre les ouvrages sans genre repéré, qui suivent globalement la courbe générale de la production, les trois genres dominants sont le roman, l'essai et la poésie, soit les genres les plus traditionnels et généralistes.

Comme on pouvait s'y attendre, l'année 1940 voit la production de livres s'effondrer. Néanmoins, dès l'année suivante, le nombre de romans publiés atteint à nouveau le total de l'année 1938, puis explose littéralement en 1942 et 1943, au point de dépasser le total de romans publiés juste avant la crise économique de $1929^{15}$. L'essai connaît le même type de courbe évolutive,

12 Ce n'est évidemment pas, par définition, un genre littéraire, mais l'étiquette permế une catégorisation qui remplit dans notre étude des fonctions similaires aux autres genres.

13 Nous avons conservé deux catégories distinctes pour « contes » et pour « nouvelles », car l'étiquette « contes » semble jouer un rôle particulier dans l'entre-deux-guerres, avec une forte présence à la fin des années 1930, comme nous avons pu le montrer dans Dozo (BjörnOlav), « La production littéraire des Belges francophones durant l'entre-deux-guerres. Examen d'un premier inventaire ", dans Mémoires du livre/Studies in Book Culture, volume $1, n^{\circ} 2,2010$ (<http://id.erudit.org/iderudit/044214ar>). Nous souhaitions vérifier que cette forte présence se confirmait au-delà de la Seconde Guerre mondiale, ce qui ne semble pas vraiment être le cas.

14 « Conférences » intègre « discours »; « contes » comprend « fables »; « essai » regroupe « manifeste », « méditation », «pamphlet » et « prose » (cette dernière mention se retrouve dans dans d'autres grandes catégories; le départ a été réalisé à partir du titre de l'œuvre); « non littéraire » reprend « bibliographie », " communication », « critique », « étude », « méthode », « guide », « histoire littéraire », « hommage », « mémoire », «monographie », « radio », «causerie » el « ouvrage de référence »; « nouvelles » englobe «prose » et « récit»; poésie réunit en plus de lui-même "aphorisme », "cantate » « chanson », «épopée », « poème(s)», « recueil » et « tract surréaliste »; « roman » inclut « cahiers », « lettres romanesques » et « littérature jeunesse »; « témoignages » incorpore « autobiographie », « biographie - souvenirs », « entretiens », " journal », « mémoires », «notes », « récit autobiographique », « récit de voyage », « reportage », « souvenirs », " texte autobiographique » et « voyage »; " théâtre » embrasse " ballet », " comédie », « drame », « fable », « mystères », « ode dramatique », " opéra » et « pièce ». Le volume de chaque sous-catégorie est très fable (généralement entre 1 et 5 occurrences), à l'exception de quelques étiquettes classiques (« poème(s) », « souvenirs », « comédie », etc.).

15 Sur l'édition durant la Seconde Guerre mondiale, voir la thèse de Finceur (Michel), Contribution à l'histoire de l'édition francophone belge sous l'occupation allemande (1940-1944), ULB, 2006. 

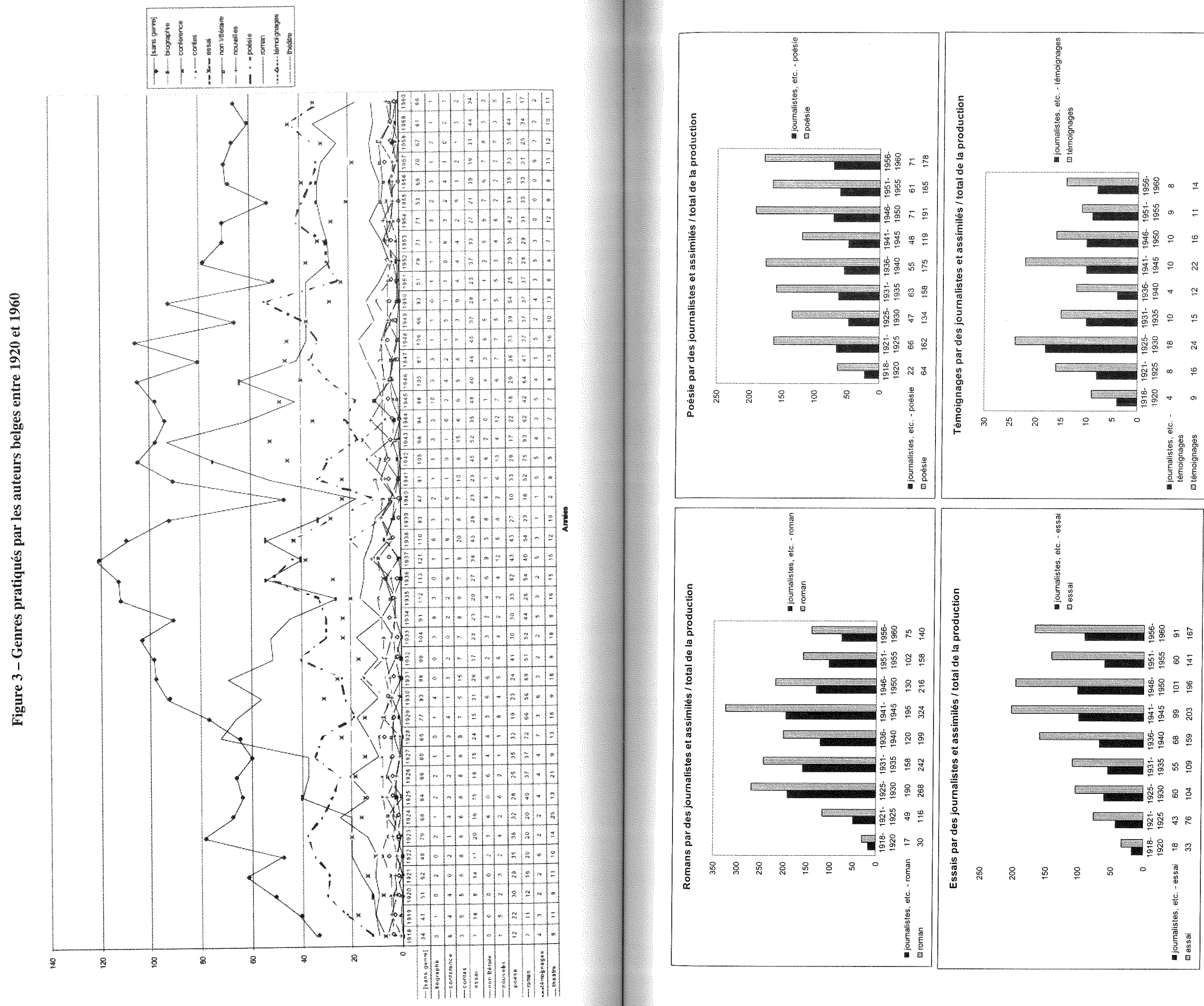

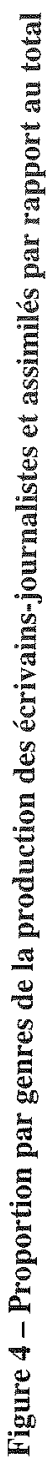

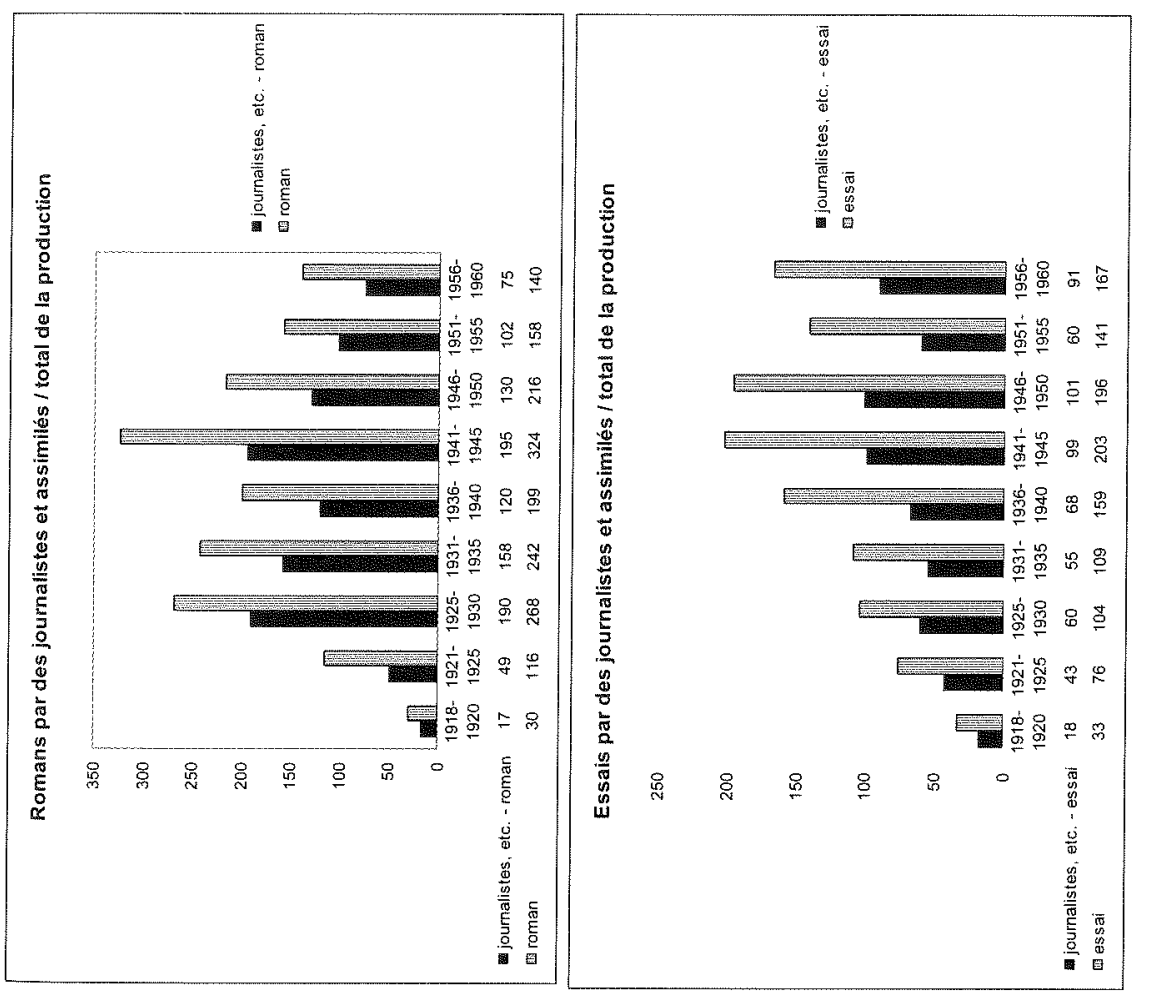

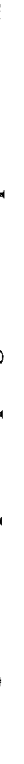


avec des chiffres moins élevés. La poésie, en revanche, reste en deçà de son nombre de publications de l'entre-deux-guerres. Elle ne retrouvera les totaux de 1936 qu'en 1950, avec l'émergence d'une nouvelle génération de poètes.

Les autres genres sont nettement moins représentés, se maintenant largement sous la barre des 20 éditions par an (sauf le théâtre, qui oscille entre 15 et 20 , avec une pointe à 25 en 1924). Le conte, genre en vogue dans les années 1930 (15 recueils en 1931, 20 en 1938), s'effondre après la guerre. Comme nous l'avions supposé dans notre article sur la production littéraire durant l'entre-deux-guerres, le conte est particulièrement en vogue durant cette période. Une étude qualitative de la production de l'époque devrait pouvoir expliquer ce succès.

Ce rapide tableau de la production totale va être comparé à la production littéraire des écrivains exerçant une profession de l'information, des arts et des spectacles. Pour plus de lisibilité, nous avons regroupé la production en tranches de cinq ans (durée de la Seconde Guerre mondiale). Nous ne représentons sur les graphiques suivants que quatre catégories : le roman, l'essai et la poésie, étant donné leur domination quantitative, et la littérature de témoignages (en ce compris les reportages, mais aussi les mémoires, les souvenirs, etc.), qui aurait pu entretenir un lien privilégié avec cette espèce particulière de littérateurs. (v. fig 4 p. 133)

Globalement, il n'y a pas de disproportion entre l'évolution de la production de ces écrivains et l'évolution générale de la production littéraire belge, ni de disproportion extrême concernant l'un ou l'autre genre privilégié. On remarque néanmoins que certains genres sont plus fréquentés par les écrivains-journalistes et assimilés, et d'autres plutôt négligés.

De manière assez attendue, on note ainsi que le roman et l'essai sont les modes d'expression littéraire favoris de cette catégorie d'écrivains, qui délaissent a contrario la poésie. Quant à la littérature de témoignage, si elle rencontre bien les intérêts des écrivains-journalistes, le lien n'apparaît pas aussi fort que prévu: en fonction des années, la proportion des titres produits par des écrivains-journalistes oscille entre un tiers et (rarement) trois-quarts, avec une moyenne légèrement supérieure à la moitié du total.

Pour se faire une idée générale de l'apport de ces écrivains à la littérature, observons la proportion de leur production par rapport au total par genre sur toute la période.
Figure 5-Production générique des écrivains-journalistes par rapport aux autres écrivains

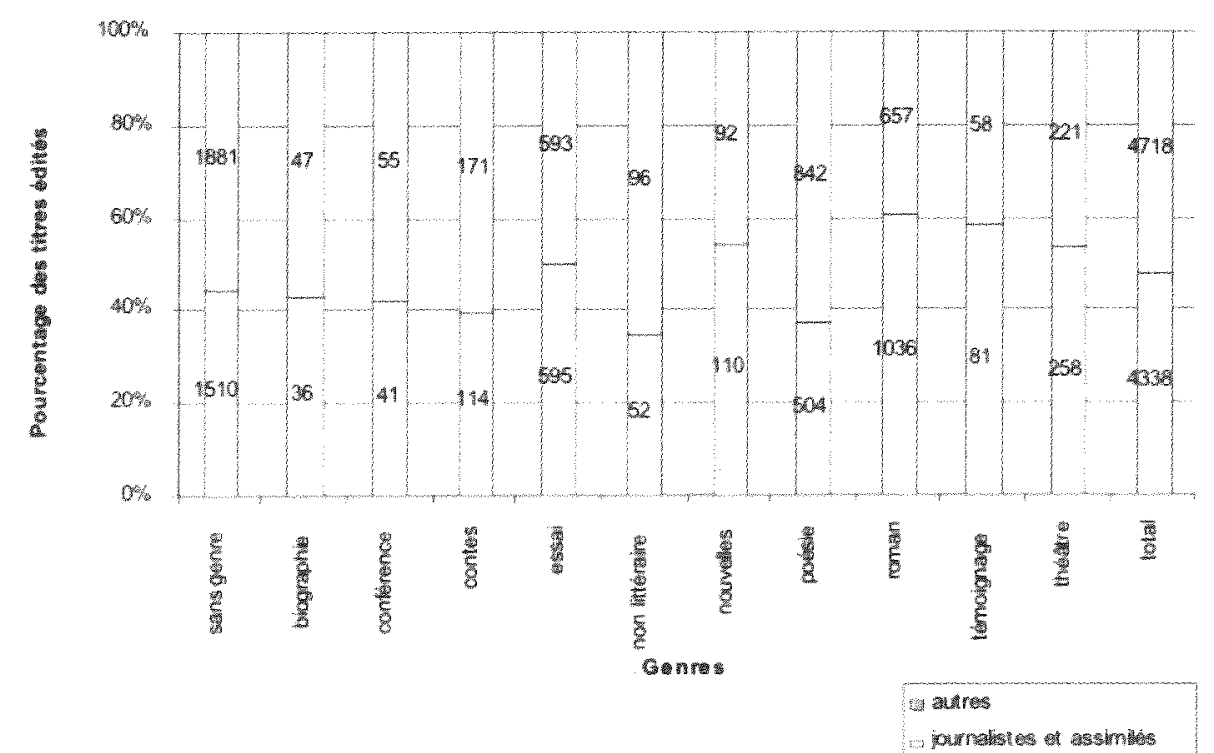

Voici la traduction du graphique en tableau, pour une comparaison plus aisée entre la production des écrivains-journalistes et assimilés, et celle des autres écrivains.

Tableau 1 - Production générique des écrivains-journalistes par rapport aux autres écrivains

\begin{tabular}{|c|c|c|c|c|c|c|c|c|c|c|c|c|}
\hline & 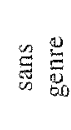 & 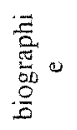 & 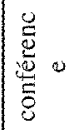 & 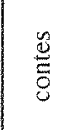 & 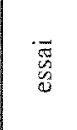 & 怘总 & 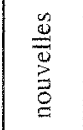 & $\begin{array}{l}\text { 要 } \\
8 \\
8 \\
0\end{array}$ & 焉 & 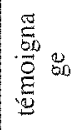 & 总 & 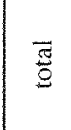 \\
\hline $\begin{array}{c}\text { Journalistes et } \\
\text { assimilés }\end{array}$ & 1510 & 36 & 41 & 114 & 595 & 52 & 110 & 504 & 1036 & 81 & 258 & 4338 \\
\hline autres & 1881 & 47 & 55 & $17 !$ & 593 & 96 & 92 & 842 & 657 & 58 & 221 & 4718 \\
\hline total & 3391 & 83 & 96 & 285 & 1188 & 148 & 202 & 1346 & 1693 & 139 & 479 & 9056 \\
\hline proportion & & & & & & & & & & & & \\
\hline $\begin{array}{l}\text { pour les } \\
\text { journalistes et } \\
\text { assimilés }\end{array}$ & 44,53 & 43,37 & 42,71 & 40,00 & 50,08 & 35,14 & 54,46 & 37,44 & 61,19 & 58,27 & 53,86 & 47,90 \\
\hline
\end{tabular}

Deux genres sont nettementen retrait: la poésie et la production non littéraire. Cette dernière est essentiellement le fruit d'universitaires, d'enseignants ou de critiques, autre grande catégorie socioprofessionnelle dont le métier s'associe aisément avec celui d'écrivain. En observant les genres privilégiés par les écrivains exerçant une profession de l'information, des arts et des spectacles (roman, témoignage, nouvelle, essai), il est raisonnable de postuler qu'ils 
favorisent une esthétique à tendance réaliste, qui correspond tendanciellement davantage à ces genres. À l'appui de cette thèse, leur désaffection pour le conte, qui s'oppose à leur attrait pour la nouvelle. Cette option réaliste serait à confirmer par une approche esthétique des œuvres.

Une remarque enfin sur la proportion numérique d'œuvres dues à des écrivains-journalistes et assimilés : fort d'un contingent de 225 auteurs sur 522 en activité durant la période considérée, soit environ $43 \%$, ils produisent 4338 titres édités sur 9056 , soit presque $48 \%$. Leur activité est donc très légèrement supérieure à la moyenne, avec plus de 19 éditions de livres par écrivain, contre un peu plus de 15 éditions de livres par écrivain belge non journaliste ou assimilé. Simenon et ses 341 éditions publiées sur la période étudiée pèse évidemment sur cette moyenne.

\section{Chez quels éditeurs publient les écrivains-journalistes?}

Étudier les éditeurs de la production des écrivains-journalistes et assimilés permet notamment de mieux cerner la légitimité et la diffusion de leur production. C'est également un indice de leur inscription plus ou moins exclusive dans le sous-champ littéraire belge.

Sur les 9056 éditions d'œuvres répertoriées au total, nous possédons des informations au sujet de l'éditeur pour 8557 éditions (pour 499 éditions d'œuvres, l'éditeur est donc manquant). Le graphique suivant présente les 46 éditeurs qui publient le plus d'œuvres de Belges francophones entre 1918 et 1960. La Renaissance du Livre domine largement le tableau (383 éditions), suivie, pour les éditeurs belges, par Les Éditions de Belgique (158) et l'Office de Publicité (156). Du côté des éditeurs français, Gallimard et la Nrf ont publié 192 éditions et Fayard 115, devant les éditions Ferenczi et fils (90). (v. fig. 6 p. 137)

Examinons la situation pour les écrivains exerçant une profession de l'information, des arts et des spectacles. Si l'on retient les 48 plus gros éditeurs d'auteurs belges, on observe différentes modifications. Disparaissent ainsi A. Dewit, Desoer, Durendal associé à P. Lethielleux (mais Durendal seul reste), Dutilleul, F. Larcier, J. Vermaut, La Revue sincère, Le Thyrse, L'Essor, Thorne, Vanderlinden. A. Dewit (58 éditions), Vanderlinden ( 77 éditions) et J. Vermaut (49 éditions) dans une moindre mesure sont les disparitions les plus surprenantes, étant donné l'abondance de leur production.

Il est intéressant de signaler que de petites maisons adossées à des revues (de poésie bien souvent) se maintiennent : Les Cahiers du Journal des Poètes, Lumière, Ça ira! publient des écrivains-journalistes. Si les premiers publient exclusivement de la poésie et constituent un des éditeurs des rares journalistes poètes, les deux suivants diversifient leur production : romans, essais, nouvelles, etc. se rencontrent dans leur catalogue.

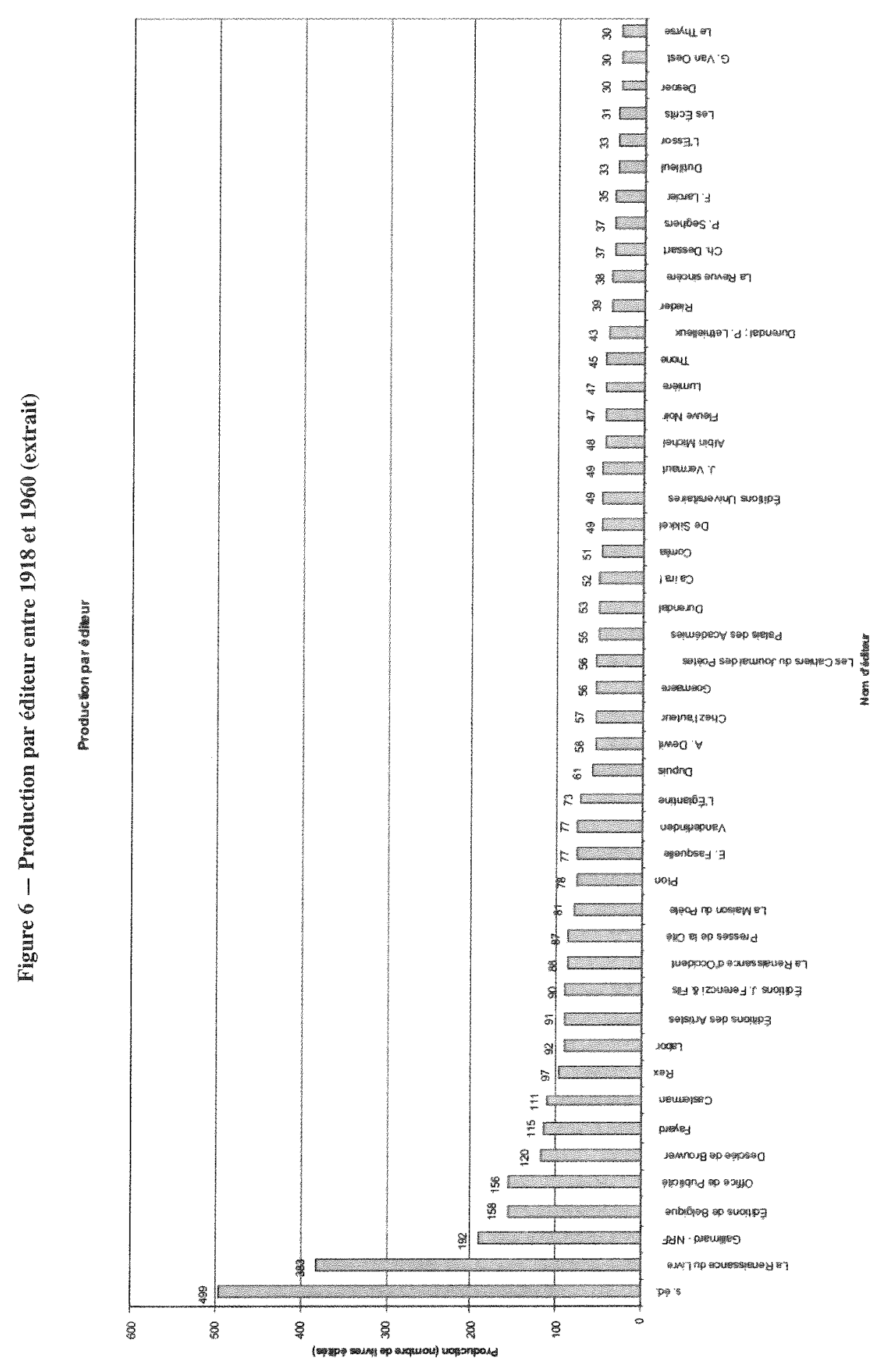


Les maisons d'édition idéologiquement engagées restent présentes également : Labor et Rieder, mais aussi Rex dans l'entre-deux-guerres.

Enfin apparaissent une série d'autres éditeurs, dont la production de titres d'écrivains belges est trop réduite pour faire partie des plus gros éditeurs à cet égard (ils publient généralement une vingtaine d'éditions ou moins), mais qui impriment suffisamment d'écrivains-journalistes et assimilés pour justifier leur présence : A. Beirnaerdt, Brépols, Denoël, Éditions Jules Tallandier, Hachette, La Vie intellectuelle, L'Audiothèque, L'Étoile, la Librairie des Champs-Élysées, la Nouvelle Société d'éditions, Prima et René Julliard. (v. fig. 7 p. 138)

Il est intéressant de s'interroger sur la proportion d'œuvres d'écrivainsjournalistes au sein des maisons d'édition les plus importantes, pour voir notamment si certaines, parmi les auteurs belges, privilégient les écrivains exerçant une profession de l'information, des arts et des spectacles. Voici le tableau des éditeurs les plus importants qui publient aussi des écrivains de cette catégorie

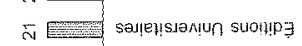

$\overline{\mathrm{N}}=$ Hessed 40

$\bar{N}=$ i

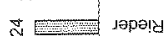

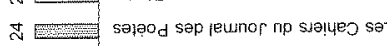

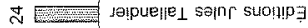

ì

I

品

$8 \rightleftharpoons$

8 = uemąse

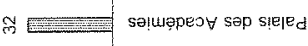

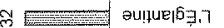

品

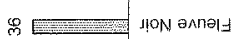

go

of

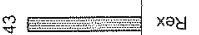

g

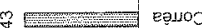

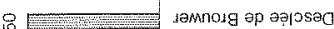

कि

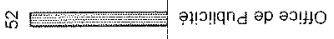

品

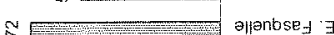

\&

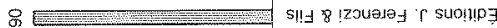

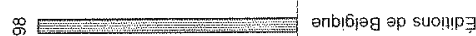

ô

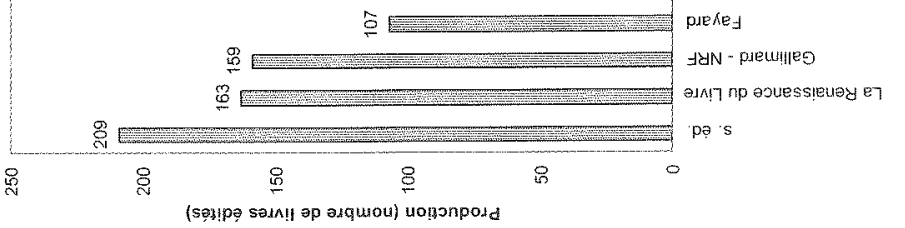

Editeurs

\section{Nb d'édit. nd d'édit. \% Nb d'écrivains- \\ total de journalistes \\ journalistes}

su catal.

Albin Michel

ça ira!

Casterman

Ch. Dessart

Chez l'auteur

Corrêa

De Sikkel

Desclée de Brouwer

Dupuis

Durendal

É. Fasquelle

Éditions de Belgique

Éditions des Artistes

Éditions J. Ferenczi \& Fils

Éditions Universitaires

Fayard

Fleuve Noir

G. Van Oest

Gallimard

Goemaere

La Maison du Poète

$\begin{array}{rrrr}48 & 35 & 73 & 9 \\ 52 & 21 & 40 & 13 \\ 111 & 30 & 27 & 15 \\ 37 & 21 & 57 & 10 \\ 57 & 27 & 47 & 13 \\ 51 & 43 & 84 & 5 \\ 49 & 30 & 61 & 16 \\ 120 & 50 & 42 & 18 \\ 61 & 43 & 70 & 8 \\ 53 & 17 & 32 & 14 \\ 77 & 72 & 94 & 5 \\ 158 & 98 & 62 & 18 \\ 91 & 39 & 43 & 18 \\ 90 & 90 & 100 & 1 \\ 49 & 21 & 43 & 13 \\ 115 & 107 & 93 & 22 \\ 47 & 36 & 77 & 1 \\ 30 & 16 & 53 & 21 \\ 192 & 159 & 83 & 26 \\ 56 & 17 & 30 & 9 \\ 81 & 30 & 37 & 15\end{array}$

9

3

15

0

5

16

8

14

5

18

8

13

22

21

26

15 


$\begin{array}{lrrrr}\text { La Renaissance d'Occident } & 88 & 40 & 45 & 18 \\ \text { La Renaissance du Livre } & 383 & 163 & 43 & 64 \\ \text { Labor } & 92 & 51 & 55 & 30 \\ \text { L'Églantine } & 73 & 32 & 44 & 20 \\ \text { Les Cahiers du Journal des Poètes } & 56 & 24 & 43 & 17 \\ \text { Les Écrits } & 31 & 16 & 52 & 11 \\ \text { Lumière } & 47 & 29 & 62 & 14 \\ \text { Office de Publicité } & 156 & 52 & 33 & 25 \\ \text { P. Seghers } & 37 & 19 & 51 & 12 \\ \text { Palais des Académies } & 55 & 32 & 58 & 17 \\ \text { Plon } & 78 & 53 & 68 & 17 \\ \text { Presses de la Cité } & 87 & 86 & 99 & 3 \\ \text { Rex } & 97 & 43 & 44 & 14 \\ \text { Rieder } & 39 & 24 & 62 & 7\end{array}$

Tableau 2 - Plus gros éditeurs publiant des écrivains-journalistes ${ }^{16}$

Les pourcentages très élevés de Fayard $(93 \%)$, des Éditions J. Ferenczi \& Fils (100\%), des Presses de la Cité (99\%) et de Gallimard s'expliquent par la présence de Georges Simenon dans leur catalogue.

D'autres auteurs pèsent aussi sur ces statistiques par leur production importante et leur attachement à une maison d'édition : Félicien Marceau pour Gallimard, Stanislas-André Steeman dans une moindre mesure pour les Presses de la Cité, etc. Pour être interprétés comme l'indice d'un lien fort entre l'éditeur et la vie littéraire belge francophone, ces pourcentages doivent être pondérés par un indice de la diversité des auteurs par éditeur. D'où la présence de la cinquième colonne : un pourcentage élevé de titres d'écrivainsjournalistes et assimilés aura plus de sens si ces derniers sont nombreux au sein de la maison d'édition.

Une dernière remarque à propos de ce tableau : on y rencontre un dernier élément notable, qui pourrait être négligeable, comme nous l'avons signalé lors de la définition du corpus, si l'écrivain concerné n'avait pas bénéficié de tant de rééditions. Il s'agit de la maison E. Fasquelle et de Maurice Maeterlinck. Celui-ci a été inclus dans le corpus en sa qualité d'écrivain professionnel ${ }^{17}$. En effet, la profession d'écrivain appartient à la catégorie des professions IAS, fondée comme on l'a dit sur l'idée d'un rapport au

16 En grasses, les pourcentages d'éditions d'écrivains-journalistes supérieurs à la moitié de la production de l'éditeur.

17 Cette qualification est, dans le cas de Maeterlinck, discutable. On pourrait plutôt parler de rentier qui consacre son temps à l'écriture. Néanmoins, le nombre d'éditions de ses ouvrages (même si certains connaissent un tirage et une diffusion confidentiels au départ) et littéraire ou plus généralement encore à l'artistique comme « gagne-pain » et qui s'est substituée au début de l'article à une définition stricte de l'écrivainjournaliste. Cette définition maximaliste n'a pas eu de conséquence notoire sur l'analyse biographique des écrivains-journalistes. Rares sont en effet ces cas particuliers, comme nous l'avons vu : ils ne viennent que peu parasiter les résultats si l'on s'intéresse uniquement aux écrivains-journalistes. Ils ne sont en effet que 3 (Maurice Maeterlinck, Henri Michaux et Charles Plisnier) sur 225 à avoir le statut d'《 écrivain professionnel » et 10 à avoir celui d' « homme de lettres », sans avoir exercé à un moment de leur vie le métier de journaliste ${ }^{18}$. De plus, le statut $d^{d}$ ' homme de lettres » cache bien souvent une absence de renseignements détaillés sur la profession exercée, qui peut se révéler être une forme de journalisme ${ }^{19}$. Tant qu'on s'en tenait à l'individu comme objet d'étude, l'écrivain professionnel ne surpondérait donc pas la catégorie des professions IAS. Néanmoins, en abordant la production littéraire en tant que telle, on change d'objet. Il est donc important de signaler qu'un écrivain professionnel à la production abondante (Maeterlinck, en somme) crée une distorsion à laquelle il fallait être attentif.

Pour en terminer avec les éditeurs, évoquons brièvement les lieux d'édition : y a-t-il un tropisme parisien chez l'écrivain-journaliste belge en matière de publication?

On dénombre environ 580 lieux d'édition dans la base de données du caEL. «Environ», parce que certains éditeurs revendiquent plusieurs villes : Paris est ainsi la coqueluche des éditeurs bicéphales belges. En fonction des éditions, ces éditeurs apparaissent parfois uniquement à Bruxelles, parfois à Bruxelles et à Paris, parfois à Paris seulement. S'il existe certains cas de déménagements effectifs du siège social, la grande majorité des adresses combinées sont liées à la légitimité littéraire de la ville-lumière. En ignorant ces petites variations, qui ne concernent finalement qu'un nombre assez restreint d'ouvrages, on constate que, entre 1918 et 1960, 3494 titres le sont à Bruxelles, 1999 à Paris et 2981 ailleurs. On ignore le lieu d'édition de 582 éditions. En ce qui concerne les écrivains-journalistes et assimilés, 1570 titres sont édités à Bruxelles, 1386 à Paris et 1155 ailleurs (le lieu est inconnu pour 227 éditions). Il apparaît donc clairement que les écrivains ayant exercé une profession de l'information, des arts et des spectacles jouent la carte parisienne pour leurs cuvres littéraires. Doit-on y voir une plus grande reconnaissance de leur art, au moins concernant quelques-uns qui produisent beaucoup ? Ce n'est pas impossible, quand on pense à Simenon et à Maeterlinck. Néanmoins, d'autres éléments peuvent jouer. On peut par exemple postuler que la circulation de leur

les prix qu'il a reçus lui ont assuré des revenus liés à l'écriture. C'est la raison pour laquelle l'étiquette d' « écrivain professionnel » n'est pas injustifiée.

18 Au total, la base reprend 26 auteurs dont la profession est à un moment de leur vie «écrivain professionnel » ou « homme de lettres ».

19 Le cas avait déjà pu être observé lors de l'examen du sous-corpus féminin. 
nom dans la presse leur sert de carte de visite auprès des éditeurs parisiens ; que leur réseau de relations intra- et extra-littéraires profite de leur profession de journaliste; que les sujets traités, plus en phase avec l'actualité, reçoivent une meilleure écoute en France; etc. Un examen plus approfondi de ce que publient ces écrivains-journalistes serait nécessaire pour poursuivre plus avant l'investigation.

\section{Dans quels journaux publient les écrivains-journalistes?}

Une dernière question s'impose concemant ce personnel littéraire spécifique : où écrivent-ils en tant que journalistes ? Une réponse précise impliquerait l'examen détaillé de la contribution de chacun à chaque périodique. Malheureusement, l'état actuel de développement de la base du CIEL ne permet pas ce type de requête. Le dépouillement des revues littéraires est encore trop lacunaire et le dépouillement des journaux n'est pas prévu de manière systématique. Nous pouvons seulement esquisser une réponse à partir du champ «participation à un périodique » de la base, champ fondé sur le collationnement des informations de la Bibliographie des Ecrivains Français de Belgique ${ }^{20}$ et d'autres sources.

Parmi tous les journaux d' information, Le Soir s'impose comme celui auquel le plus d'écrivains-journalistes ont participé. Ils sont 55 à avoir appartenu à la rédaction du journal. Suivent ensuite, dans la presse d'information, L'Indépendance belge (35), La Nation belge (28), La Meuse (21), Le Peuple (18), Pourquoi pas ? (17), La Libre Belgique (16), L'Étoile belge (16), La Gazette de Charleroi (14), Le Petit Bleu (12) et Le Xx siècle (12). Sept écrivains-journalistes ont écrit pour La Gazette, Pan, Le Soir illustré. Six pour L'Avenir du Luxembourg et La Dernière Heure.

En ce qui concerne la presse parisienne, neuf ont travaillé pour Le Monde, six pour Le Figaro, cinq pour Le Figaro littéraire, La Quinzaine littéraire, L'Humanité et L'Express.

Enfin, au niveau des revues littéraires, la plus fréquentée est Le Thyrse (38 collaborateurs), suivie de près par Le Journal des Poètes (33) et La Revue générale (32). De très nombreuses autres revues apparaissent dans la liste, mais il serait beaucoup plus utile de revenir sur le sujet une fois que le dépouillement de ces revues aura été effectué de manière systématique.

20 Brucher (Roger) puis Detemmerman (Jacques), dir., Bibliographie des Écrivains Français de Belgique, Bruxelles, Palais des Académies, 1958-1988, 5 volumes.

\section{Conclusion}

À partir des éléments présentés dressons un portrait type de l'écrivainjournaliste belge francophone actif à un moment entre 1918 et 1960. Tout d'abord, ce portrait concerne un écrivain sur trois. L'écrivain-journaliste typique est de sexe masculin, a deux chances sur trois d'exercer en plus une autre profession à un moment de sa vie, en particulier dans l'enseignement ou dans la fonction publique. Il fréquente les lieux de sociabilité littéraire : il existe par exemple une probabilité de plus de $50 \%$ pour qu'il soit membre de l'Association des Écrivains Belges. Il publie des romans, des essais, des nouvelles; des récits de témoignage bien sûr, mais il n'en a pas l'exclusive. Peu de poésie ou de contes. Il se fait publier à Paris, chez Fayard, Gallimard, Albin Michel ou Plon, ou, à défaut, à Bruxelles, à La Renaissance du Livre, aux Éditions de Belgique, chez Desclée de Brouwer ou chez Labor. Il publie beaucoup, souvent plus que les autres écrivains belges. S'il privilégie Paris pour la littérature, il travaille comme journaliste plutôt dans des quotidiens belges.

L'écrivain-journaliste tel que décrit par la statistique n'a évidemment jamais existé. II n'est qu'un type, un individu modal actualisé différemment par chaque avatar. L'intérêt de ce profil-type est d'avoir maintenant un étalon auquel comparer le profil individuel de chaque écrivain-joumaliste. Chaque étude de cas pourra s'appuyer sur ce portrait statistique, afin de repérer les dissonances et les conformités de son objet face à cet « écrivain-journaliste modal » que nous avons pu construire.

Pour aller plus loin et construire des types plus spécifiques d'écrivainsjournalistes, qui permettraient des oppositions structurantes entre profils, il faudrait multiplier les modalités descriptives (par exemple les engagements politiques et sociaux) et croiser ces différentes modalités pour vérifier les corrélations entre celles-ci suggérées par le sens commun. Un écrivainjournaliste travaillant dans un journal de gauche publie-t-il nécessairement dans des maisons d'édition ou des revues littéraires progressistes? Peut-on observer du point de vue politique une rupture entre les pratiques journalistique et littéraire? Y a-t-il des études qui prédisposent au journalisme? Peut-on dégager des liens privilégiés entre certains établissements scolaires et certains journaux ? Cet article n'a pas épuisé toutes les questions sur le sujet, loin de là ; cependant, celles-ci nécessiteraient des données supplémentaires. L'enrichissement de la base du CaEL pourrait aboutir à différents approfondissements complémentaires et ainsi à une connaissance fine de profils d'écrivains-journalistes concurrents. 